\title{
DYNAMIC BEHAVIOUR OF THE LOADS OF PODDED PROPELLERS IN WAVES: EXPERIMENTAL AND NUMERICAL SIMULATIONS
}

\author{
Patrick Queutey \\ Jeroen Wackers \\ Alban Leroyer \\ GanBo Deng \\ Emmanuel Guilmineau \\ Michel Visonneau \\ LHEEA, CNRS UMR 6598 \\ Ecole Centrale de Nantes, 1 rue de la Noë \\ BP 921044321 Nantes Cedex 3, France
}

\author{
Gerco Hagesteijn \\ Joris Brouwer \\ MAritime Research Institute Netherlands (MARIN) \\ Haagsteeg 2 \\ P.O. Box 28 \\ 6700 AA Wageningen, The Netherlands
}

\section{ABSTRACT}

The paper focuses on the hydrodynamic flow around a ship with pods in waves and compares the results of an experimental campaign with numerical simulations conducted during the EUfunded STREAMLINE project. It was the first project for which the effect of waves on cavitation and ventilation was explored in both experimental and numerical ways for a ship with pods. The measurements were carried out in MARIN's Depressurized Wave Basin (DWB) with a fully instrumented podded ship model, in sailing condition, in waves and depressurised conditions. In this way, the correct representation of cavitation and possible ventilation bubbles and vortices is ensured, resulting in a correct physical behaviour. The discretisation of the Reynolds-Averaged Navier-Stokes Equations (RANSE) is based on the unstructured finite-volume flow solver ISIS-CFD developed by ECN-CNRS. An essential feature for full RANSE simulations with this code is the use of a sliding grid technique to simulate the real propeller rotating behind a ship hull. The computational study in operational service conditions considered here has been conducted to evaluate the instantaneous flow distribution around the podded propellers and to analyse and to compare the unsteady behaviour of the forces induced by the rotating propeller in waves with the measurements from omnidirectional propeller loads as well as the blade forces and moments. The computational study has been done in model and full scale to evaluate the scale effects.

\section{INTRODUCTION}

A podded propeller (pod) as shown in Figure 1, consists of an electric motor which directly drives the propeller. The propeller can be pulling (in front of the pod) or pushing (behind the pod). The benefits of pulling propellers, streamlining of the support housings and the consequent improvement in course stability and manoeuvrability have been well demonstrated by several suppliers with fuel savings of the order of $5 \%$ to $20 \%$ relative to pushing azimuthing thrusters. Pod units already exist for most kinds of ship, with cruise liners being the most frequent users. One of the big advantages of using pod units is the flexibility in general arrangement of the ship. In the framework of the electric-ship concept, new pod driven propulsor designs will enable the maximum benefits of electric propulsion to apply to a wider range of hull forms and speeds.

Regarding the propeller (as a mechanical system), the breakthrough represented by the introduction of the podded propulsion (years 1990s for first systems in the market) was also supported by several research projects. Among these projects the EUfunded STREAMLINE project, partly funded by the 7th Framework Programme of the European Commission (FP7), is the first project for which the effect of waves on cavitation and ventilation was explored in both experimental and numerical ways for a ship with pods.

A major objective of the STREAMLINE project is to inves- 


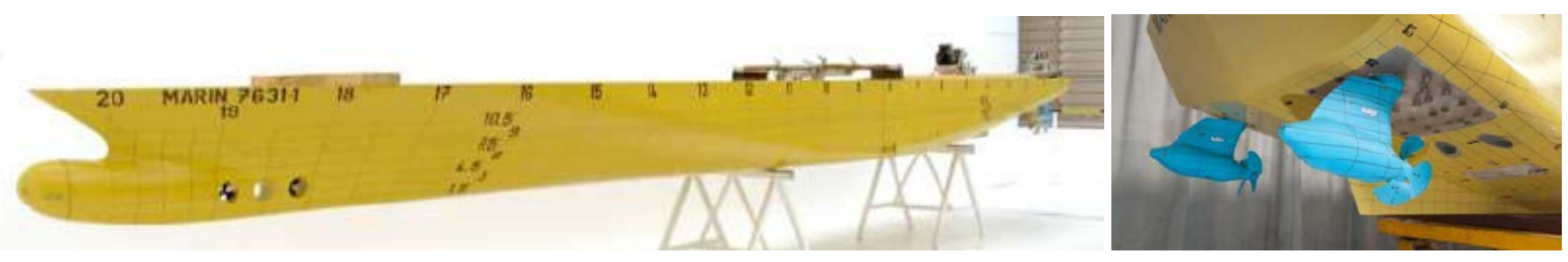

FIGURE 1. SHIP MODEL WITH PODDED PROPELLERS.

tigate methods to fully optimise current state-of-the-art (SoA) systems including conventional screw propeller systems and pods. The key here is exploitation of advanced Computational Fluid Dynamics (CFD) methods to pursue improvements without dramatic vessel configuration changes. It is also worthwhile to examine the possibility of using pod units in new locations on the hull, while previously the high risks of ventilation could not be properly assessed. To allow more accurate analysis and improved design of propulsors, these enhanced CFD tools have to demonstrate their capability in providing a detailed analysis of the propulsor in the distorted wake field produced by the aftbody of the ship in situations as close as possible of real and full-scale operating conditions. In order to achieve this goal, it is mandatory to evaluate the predictive performance and accurate modelling of the numerical simulations involving more and more complex geometries and flow conditions. Validation with Experimental Fluid Dynamics (EFD) model tests results is therefore important.

The measurements were carried out in MARIN's Depressurized Wave Basin (DWB) with a fully instrumented podded ship model, in sailing condition, in waves and depressurised conditions. In this way, the correct representation of cavitation and possible ventilation bubbles and vortices is ensured, resulting in a correct physical behaviour. A 6-component transducer was used for measuring the omnidirectional propeller loads, while a 5-component transducer was used for measuring 2 blade forces and 3 blade moments. At the same time synchronised high speed video recordings were made to acquire insight in the occurring ventilation phenomena.

If a RANSE CFD based method is used to compute the viscous flow around the ship only, the propeller can be replaced by an equivalent body force acting in the r.h.s of the momentum equations. Improved solution can be obtained by coupling a propeller performance code based upon the Boundary Element Method (BEM) theory for the potential flow around the real propeller to the RANSE CFD solver. The latter requires the development of a special interface to extract the effective wake from RANSE solution and to produce a body force field calculated by a propeller performance code. A cost-effective preliminary design can then be achieved with a RANSE/BEM hybrid model as it was demonstrated during the STREAMLINE project in calm water conditions [1-3].

Owing to the enhanced computational resources and to move a step forward, the use of full RANSE simulations of the freesurface flow around a ship with its rotating propeller in a unique numerical framework can be considered now. Moreover, the prediction of ventilation requires the use of a full RANSE approach [4]. A common practice to take into account the relative motions of multiple bodies is to manage multiple domains such as the overlapping technique $[5,6]$ and the sliding grid technique $[1,7-9]$ to allow a part of the grid (containing the propeller) to rotate within the main part of the grid, while keeping a connection between the two parts. Nevertheless, the cost is still prohibitive to be used as a matter of routine in naval architecture design offices if we consider for instance, the challenging computation of a self-propelled ship in seakeeping situations involving severe motions and multiple time scales with complex waves.

The aim of the paper is to demonstrate that SoA full RANSE flow simulations allow a careful assessment of claimed design performance. The computational study in operational service conditions considered here has been conducted to evaluate the instantaneous flow distribution around the podded propellers. It has also been used to analyse and compare the unsteady behaviour of the forces induced by the rotating propeller in monochromatic waves with the measurements. This computational study has been performed in model (MS) and full scale (FS) conditions to evaluate the scale effects. It is outlined that the CFD to EFD comparisons correspond to experiments in atmospheric condition above the free-surface since the flow solver is not able to carry out a simulation involving a depressurization scenario. The value of this case is not only in the averaged global variables, as for ship's resistance, but in the dynamics involved. All six components of the forces and the dynamic trim and sinkage have been compared with good agreement with the experimental signals. This indicates that the CFD simulation is able to predict the main physical behaviour of such complex flows.

\section{GEOMETRY AND MAIN PARTICULARS}

The selected model is a 25.33 scaled model of a cruise liner ship, however with a more V-shaped after-body. This makes it 
more comparable with other ship types, and reduces to a large extent the risk of slamming which was regarded to be unfavourable for CFD calculations, reducing the suitability of a benchmark case. The overall view of the experimented ship model with pod units is given with Figure 1. Table 1 contains the main geometrical characteristics of the hull.

The propeller design has been selected by MARIN and represents a typical modern propeller design with four blades. The diameter $D$ is $5.38 \mathrm{~m}$. The selected POD was one of the standard Rolls-Royce Mermaid designs. The propeller axis is inclined with an angle of $7.5^{\circ}$ above the main axis of the ship in even keel position.

\section{EFD: EXPERIMENTS IN MARIN'S DEPRESSURIZED WAVE BASIN}

Experience [10] showed that the estimation of risk of ventilating propellers in traditional model scale experiments under atmospheric conditions varies from that of depressurized conditions. Moreover, entrapped air pockets are proven to behave differently at different scales [11]. Therefore, better predictions are achieved in depressurised towing tanks, where the ambient pressure is also scaled down according to Froude's law of similitude.

MARIN's Depressurized Wave Basin (DWB) is the only facility in the world that is able to generate waves in a depressurized towing tank. This ensures correct representation of the pressure inside the enclosed ventilation bubbles and vortices, resulting into more correct physic behaviour.

Measuring on phenomena which are very short in duration like ventilation can be very difficult. The dynamic range of the measurement device plays a dominant role in the ability to distinguish high frequent effects. See [12] for an elaborate explanation of the problem at hand. To ensure the registration of all phenomena or as much as possible during a ventilation event, it is aimed to have the natural frequency of the measurement system as high as possible. Based on past experience, it was decided a single blade should be instrumented and a new multi-component

TABLE 1. MAIN PARTICULARS OF THE SHIP

\begin{tabular}{lcc}
\hline & MS & FS \\
\hline Lenght between perpendiculars, $L_{P P}[\mathrm{~m}]$ & 9.179 & 232.500 \\
Lenght between perpendiculars, $L_{W L}[\mathrm{~m}]$ & 9.400 & 238.101 \\
Draught moulded on FP and AP, $T_{F}, T_{A}[\mathrm{~m}]$ & 0.308 & 7.80 \\
Displacement volume moulded $\nabla\left[\mathrm{m}^{3}\right]$ & 2.247 & 36527 \\
Wetted surface area bare hull $S_{W}\left[\mathrm{~m}^{2}\right]$ & 13.16 & 8445 \\
\hline
\end{tabular}

force-transducer needed to be developed. This way, a maximum natural frequency for the measurement device could be achieved, while asserting enough sensitivity of the transducer.

The starboard propeller was fit with the single blade forcetransducer or key-blade. Synchronised camera recordings were made with normal speed cameras and high speed cameras. Since the starboard propeller contained the 'key-blade' this was the propeller of main interest and where the cameras were aimed at. The other propeller at portside was fitted with an existing multicomponent force-transducer. This transducer has 6 degrees of freedom but was designed for use in a full propeller. While successfully used to measure blade loads in the past, the natural frequency of the setup would have been compromised if used for another key-blade measurement setup. Therefore the portside propeller measurements consist of multiple degrees of freedom forces, but do not contain as high frequency data as the 'keyblade' at starboard. During development of the multi-component force-transducer for the key-blade, it was decided to sacrifice one component, being the centrifugal force component. Therefore the key-blade transducer became a 5 component transducer instead of 6 .

Finite element calculations were used to determine the first natural frequency of the key-blade and its transducer. The first natural frequency is used to identify the frequency that still can be measured. Measurements around the natural frequency are likely to be exaggerated. Above those frequencies, the setup acts like an analogue low-pass filter, obscuring any measurements that might be of interest. To prevent exaggerated measurements around the natural frequency, filtering is applied to the measured signals during post-processing of the data.

The light loading condition of the vessel was used in combination with a series of wave periods, with the highest chance of ventilation. The model itself was free to heave and pitch, while the surge motion was controlled by a linear motor which was programmed to re-enact a damper-spring system as if the model was being pulled by soft springs. The usage of a linear motor allowed for quick changes of the systems behaviour and speed adaptive friction correction.

A series of tests were carried out which started as a systematic matrix, which was deformed during the execution of the tests to capture sufficient conditions in which ventilation occurred. Tests with interesting events were carried out both in depressurized and atmospheric conditions. During all measurement runs it was aimed to capture normal video and high speed video recordings. However, due to the relatively short buffer length of the high speed video and the large data sizes created by it, it was not possible to captures all events on high speed video. The combination of high speed video with high frequency transducer signal measurements, enables a unique and detailed investigation into the development, the growth and stability of ventilation phenomena.

The studied conditions were mainly looking a the inception 


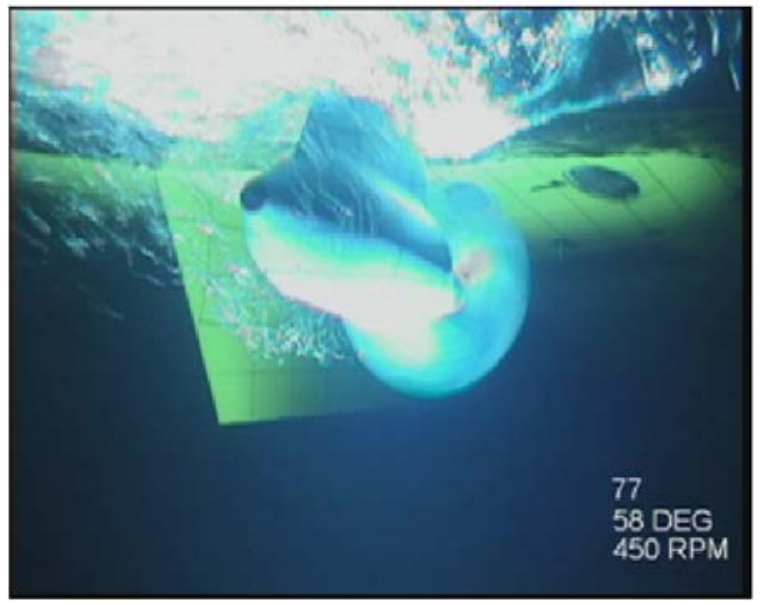

FIGURE 2. VENTILATION EVENT IN MARIN'S DEPRESSURIZED WAVE BASIN

of ventilation and cavitation. Typicially the inception of cavitation is due to decreasing hydrodynamic pressure when the water surface is moving down in a wave trough. The cavitation is only present on the top of the blades passing through the upright position. At the same time, the water surface is start to ventilate. Air, though depressurised, is being caught by the propeller and a ventilation event is started, resulting in to a caputure of the air into the downward going tip vortex. Figure 2 shows the air being thrown out aft of the pod at this moment in time.

\section{CFD: THE ISIS-CFD FLOW SOLVER}

ISIS-CFD, available as a part of the FINE ${ }^{\mathrm{TM}} / \mathrm{Marine}$ computing suite, is an incompressible unsteady Reynolds-averaged Navier-Stokes (URANS) method. The solver is fully implicit, based on the finite volume method to build the spatial discretisation of the transport equations. Surface and volume integrals are evaluated according to second-order accurate approximations and the unstructured discretisation is face-based. Time derivatives are evaluated using three-level Euler second-order accurate approximations. While all unknown state variables are cellcentred, the systems of equations used in the implicit time stepping procedure are constructed face by face. Fluxes are computed in a loop over the faces and the contribution of each face is then added to both cells sharing the face. This technique poses no specific requirements on the topology of the cells. Therefore, the grids can be completely unstructured; cells with an arbitrary number of arbitrarily-shaped faces are accepted.

Pressure-velocity coupling is obtained through a Rhie \& Chow SIMPLE type method: in each time step, the velocity updates come from the momentum equations and the pressure is given by the mass conservation law, transformed into a pressure equation. In the case of turbulent flows, transport equations for the variables in the turbulence model are added to the discretisation. Free-surface flow is simulated with a multi-phase flow approach: the water surface is captured with a conservation equation for the volume fraction of water, discretised with specific compressive discretisation schemes, see [13].

The method features sophisticated turbulence models: apart from the classical two-equation $k-\varepsilon$ and $k-\omega$ models, the anisotropic two-equation Explicit Algebraic Stress Model (EASM), as well as Reynolds Stress Transport Models, are available, see $[14,15]$. Time-integration of Newton's laws for the 6 degrees of freedom of ship motion is combined with analytical weighted or elastic analogy grid deformation to adapt the fluid mesh to the moving ship [16].

An essential building block for the simulation of ship propellers is a sliding grid technique, which allows a part of the grid (containing the propeller) to rotate within the main part of the grid, while keeping a connection between the two parts. Sliding grid methods for unstructured grids, such as we use [7-9], are far more complicated than for structured grid solvers. Among others, the connection between the two subdomains of the grid has to be reconstructed often, as it does not follow a regular pattern. Also, it is not easy to ensure flux conservation over the interface. We have chosen a connection between the domains that does not explicitly guarantee conservation; it is based on connectivities between the cells and faces on the interface that mimic as closely as possible the connectivities for all other cells in ISIS-CFD.

Parallelization is based on domain decomposition. The grid is divided into different partitions; these partitions contain the cells. The interface faces on the boundaries between the partitions are shared between the partitions; information on these faces is exchanged with the MPI (Message Passing Interface) protocol. The essential point is that all fluxes on a face can be constructed from the states and gradients in the centres of its two neighbour cells, plus the positions of these centres. In our domain decomposition approach for parallel computing, this neighbour cell information is the only data which is exchanged over the interface faces. We do not explicitly split the grid into two parts by assigning a number of processors to each sub-domain and partitioning each sub-domain separately. Instead, the grid can be arbitrarily spread over the processors, a 'colour' is assigned to each cell to indicate to which sub-domain it belongs. This gives the flexibility to run the code on a single processor and also to perform grid refinement; when a part of the grid is refined, the balance would be lost if each sub-domain were assigned to a constant number of processors. With our approach, we can redistribute freely $[8,9]$.

\section{NUMERICAL CASE}

This section is devoted to the description of one selected case for seakeeping conditions in head waves. The choice of the computational domain, boundary conditions and grid generation 
is first presented followed by a description of the numerical strategy, settings and CPU cost on an IBM x3750 cluster $(2.7 \mathrm{GHz} 8$ cores quad-processors). For reasons of symmetry, only half of the hull is considered. Then only one propeller with its pod unit is modelled.

\section{Description}

Among the large amount of test data collected in experiments, one seakeeping condition in head waves has been retained as interesting case for CFD analysis and assessment and the conditions are listed in Table 2. This selected case in atmospheric condition above the free-surface is quite challenging as it was the first time such a simulation was conducted with the flow solver. The work achieved in blind conditions, with regards to the experiments, was at the forefront of the full RANSE simulations conducted in the project. It involves interaction of the freely moving ship in waves with the rotating propeller. All the ingredients of the flow solver are involved : free-surface capturing, resolution of the Newton's law to couple the forces induced by the flow and the motions of the ship, the sliding grid approach to deal with the rotating propeller, the mesh deformation technique to adapt the grid with the ship motions including the attached rotating propeller.

The grid is generated with HEXPRESS ${ }^{\mathrm{TM}}$, the automatic hexahedral grid generator for unstructured grids. The FS computational domain extension in metres is $[-500,500] \times[0,500] \times[-$ 250,50 ] if the ship is between $0 \mathrm{~m}(\mathrm{AP})$ and $232.5 \mathrm{~m}$ (FP). In this initial condition, the propeller centre is located $4.4 \mathrm{~m}$ below the free surface level at rest corresponding to an initial submergence ratio $h / R$ of 1.636, if $h$ is the distance of the propeller centre to the free surface and $R$ is the propeller radius $2.69 \mathrm{~m}$. The vertical target cell size at the vicinity of the air/water interface is about $20 \mathrm{~cm}$ far from the hull $(8 \mathrm{~mm}$ in MS) and less than $10 \mathrm{~cm}$ (less than $1 \mathrm{~mm}$ in MS) in the propeller region. Two sub-domains are used: the first one attached to the hull including the pod unit and a second one attached to the rotating propeller, the communication between the two being performed across a cylindrical boundary according to the sliding grid communication strategy.

On the hull, pod and propeller, grid stretching is applied at the vicinity of the walls with the first layer thickness of $4 \mathrm{~mm}$ to assume a targeted $\mathrm{y}^{+}$value of 300 in FS (30 in MS), suitable for the wall-function approach for turbulent flow computations. At the inlet and outlet, supposed to be far enough from the hull, a prescribed velocity field at rest is applied while a prescribed constant pressure is used on the top and a prescribed hydrostatic pressure on the bottom. A refined grid distribution is used between the inlet and about one half ship length past in the wake in order to have a correct transport of the numerical wave generated at the inlet with a prescribed (unsteady) velocity and volume fraction distribution derived from a first order potential Stokes waves. In FS, the vertical thickness of that region is $3.8 \mathrm{~m}$ above

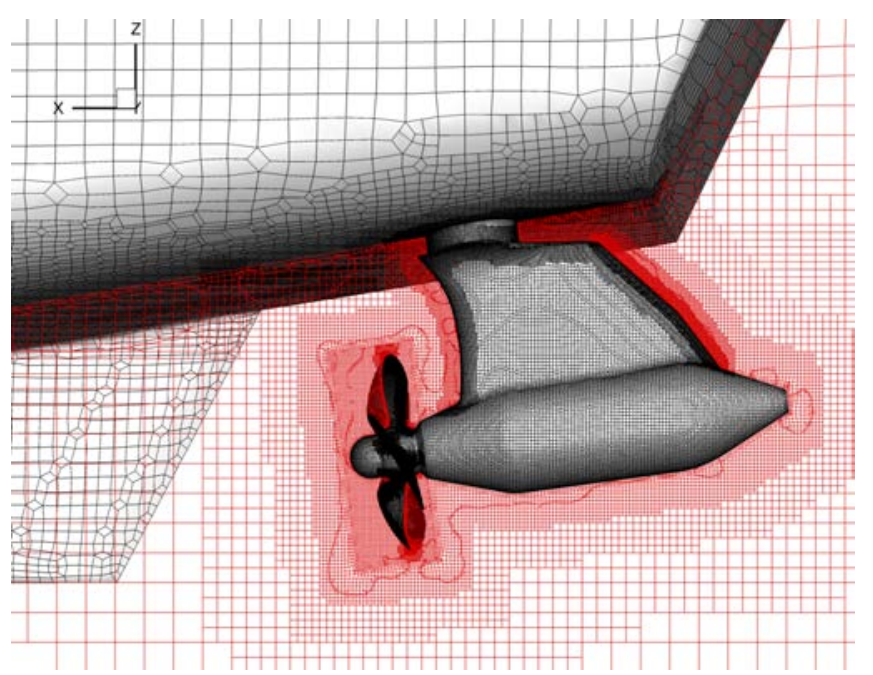

FIGURE 3. FS GRID: DETAILS ON SURFACES AND IN A LONGITUDINAL CUT PLANE PASSING THROUGH THE PROPELLER CENTRE (VISCOUS LAYERS NOT SHOWN).

and below the free surface level at rest and the longitudinal grid spacing inside is about $1.6 \mathrm{~m}$ ( 120 cells per wave length) and the vertical resolution is about $20 \mathrm{~cm}$ ( 20 cells between crests and troughs). A prescribed constant pressure is used on the top (air) and a prescribed hydrostatic pressure on the bottom. The resulting grid contains $6.1 \mathrm{M}$ cells with $2.01 \mathrm{M}$ cells in the rotating sub-domain and $4.08 \mathrm{M}$ cells in the other sub-domain (hull and pod unit). Figure 3 shows grid details on the surfaces and in a longitudinal cut plane passing through the propeller centre. For the sake of clarity, the thin viscous layers are not shown.

\section{Settings}

The $k-\omega$ SST turbulence model is used for the simulations with the wall-function approach on the walls. The processor loading retained is about $150 \mathrm{~K}$ cells per processor and the grid is partitioned into 40 blocks. The computation is run unsteady in two steps according to the dynamical parameters listed in Table 2: The numerical settings and the way the simulation is conducted in FS are as follows:

Step 1 - Starting from $t=0 \mathrm{~s}$ at rest (both advancing ship speed and propeller speed of rotation), the ship accelerates to the prescribed velocity $\mathrm{V}=5.6589 \mathrm{~m} / \mathrm{s}$ (11 knots) following a quarter-sinusoidal law until $\mathrm{t}=130 \mathrm{~s}$. In the same time, the propeller attached to hull is rotating to the prescribed revolution rate $89.4 \mathrm{rpm}(9.362 \mathrm{rad} / \mathrm{s})$ following an acceleration law from a quarter-sinusoidal law until $\mathrm{t}=10 \mathrm{~s}$. During that phase the time step is $\Delta \mathrm{t}=0.1 \mathrm{~s}$ adapted to the resolution of the numerical wave generation and transportation corresponding to slightly more than 60 time steps during one encountered period $T e=8.2738 \mathrm{~s}$. With such a time step the propeller achieves a complete rotation in nearly 7 time steps only 
( $T p=0.67114094 \mathrm{~s})$. The CPU cost for that step is about $4 \mathrm{~h}$ for 1500 time steps with 40 partitions (effective CPU cost of about 63 days) until $t=249.1 \mathrm{~s}$. Since the computation involves ship motions and interaction with the waves, this step is significantly more time-consuming in comparison with a classical resistance computation. In that case about 22 waves encounters have been computed.

Step 2 - The previous computation is restarted at $\mathrm{t}=249.1 \mathrm{~s}$ with a time step $\Delta \mathrm{t}=0.003355705 \mathrm{~s}$ to resolve the hull-propeller-wave interaction. One rotation of the propeller is achieved in 200 time steps. During that phase, 5474 time steps are used, corresponding to the resolution of about 27 rotations of the propeller. The CPU cost of that step is about $75 \mathrm{~h}$ (effective CPU cost of about 125 days) to go until $\mathrm{t}=267.47 \mathrm{~s}$, corresponding a duration in real time of $18.37 \mathrm{~s}$. This means about 2.2 computed encountered periods but nearly 30 propeller revolutions (about 12.3 revolutions per encountered wave period). Only 2.2 encounters have been computed due to our CPU limitations in this part but the unsteady signals of the forces seem reasonably well established.

\section{RESULTS}

This section is divided into three parts. The first one deals with a general description of the flow field and ship motion in waves. The second part concerns the dynamics of the forces with scale effects. The section ends with the finding of correlations between the flow field, motion in wave, and propeller thrust.

\section{Flow field}

Figure 4 presents close-up views of the computed freesurface deformations and ship motions in the propeller region at four significant time instants during on encounterd period Te. Although not shown, strong interaction is noticed in the fore region with noticeable wave breaking as the bow moves up and down.

The dynamics of the trim and sinkage are plotted in Figure 5 and compare the computed degrees of freedom of the FS ship with the measurements (extrapolated from MS to FS). The signals have been synchronized in time based upon the last but one maximum value of the pitch angle at 258.134s: see dotted vertical line in figure 5(a) corresponding to mark "0". Time resolved Step 2 starts at about $250 \mathrm{~s}$ (blue vertical dotted line). The indicated time instants are the same as those used for the computed snapshots in Figure 4. As a whole the agreement is good between the computation and the measurements however it appears that the kinematics of the theoretical wave used in the computation at the inlet generates an encountered period Te of $8.07 \mathrm{~s}$ compared to the theoretical value of $8.27 \mathrm{~s}$ and to the experimental value of $8.29 \mathrm{~s}$. The computed averaged amplitude of the vertical displacement of the centre of gravity is $1.26 \mathrm{~m}$ and measured 1.24 $\mathrm{m}$. The corresponding amplitude on the pitch is $1.615^{\circ}$ for the

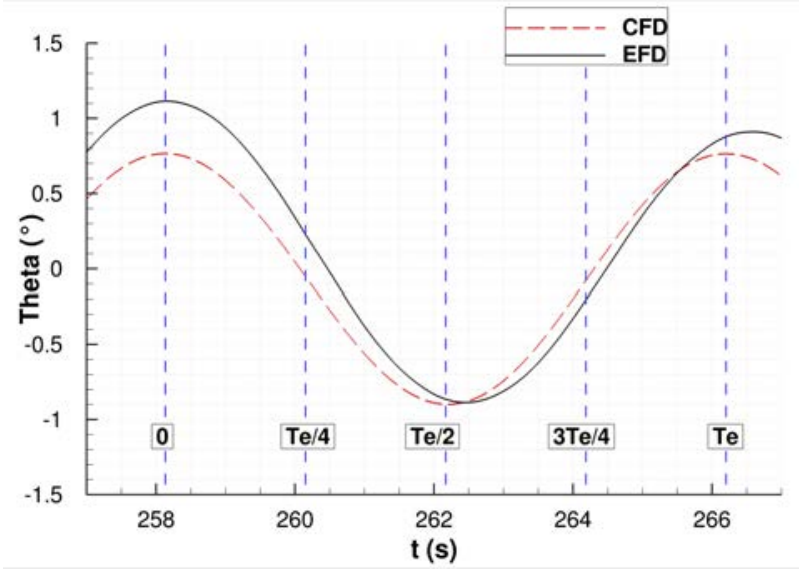

(a) Pitch

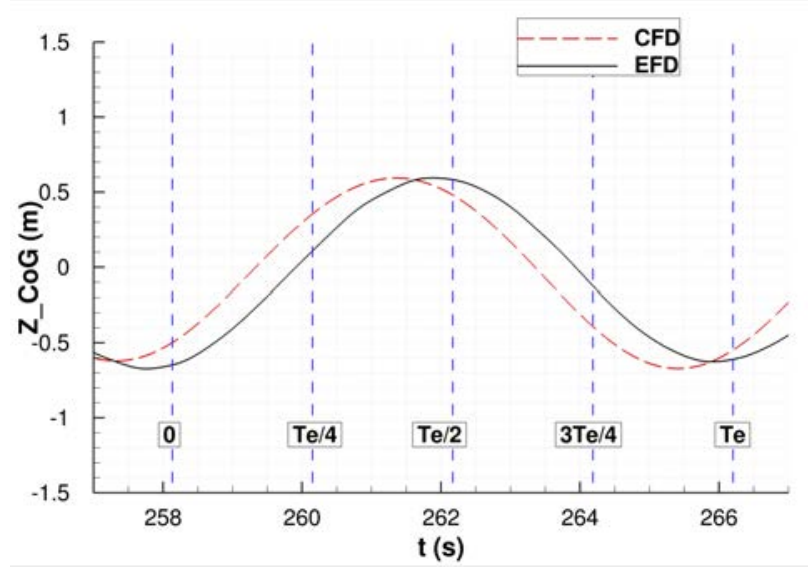

(b) Heave

FIGURE 5. TIME HISTORIES OF THE PITCH AND HEAVE OF THE SHIP. EFD: SOLID LINE, CFD: DASHED LINE.

computation compared to the measured $1.70^{\circ}$.

Considering the computed ship motion and evolution of the position of the wave trough, it appears that the time instant when the submergence ratio reaches a minimum of about 1.36 is around 0.81Te (between $\mathrm{t} 3$ and $\mathrm{t} 0$ ). Although the submergence ratio is low at that time instant, no ventilation events have been detected in the simulations in model or in FS. It is outlined that the pitch and heave signals are in opposition of phase and the evolution of the wave trough in the propeller region is such that the combination of the ship motions and the dynamics of the waves is not favourable to ventilation: as an example, at Te/2 $(\mathrm{t}=\mathrm{t} 2)$, the ship $\mathrm{CG}$ is in the highest vertical position while the pitch angle value at the lower such that the propeller remains submerged between a wave trough and a wave crest. As stated in experiments, indeed the ventilation measured is scarce. It is assumed that this process is very sensitive to the exact height of 
TABLE 2. DYNAMIC PARAMETERS OF THE MODEL AND FS SHIP. THE ORIGIN OF Z IS AT THE KEEL.

\begin{tabular}{lcc}
\hline Quantity & MS & FS \\
\hline Lpp & $9.17884 \mathrm{~m}$ & $232.5 \mathrm{~m}$ \\
Draught & $30.7935 \mathrm{~cm}$ & $7.8 \mathrm{~m}$ \\
Centre of gravity $(\mathrm{X}, \mathrm{Y}, \mathrm{Z})$ & $(4.155419 \mathrm{~m}, 0 \mathrm{~m}, 0.1724686 \mathrm{~m})$ & $(105.2568 \mathrm{~m}, 0 \mathrm{~m}, 4.3686 \mathrm{~m})$ \\
Mass & $2230.792 \mathrm{~kg}$ & $36254730 \mathrm{~kg}$ \\
Moment of inertia $I_{y y}$ & $13046.15 \mathrm{~kg} \cdot \mathrm{m}^{2}$ & $13697640 \mathrm{~kg} \cdot \mathrm{m}^{2}$ \\
Ship speed & $1.12447 \mathrm{~m} / \mathrm{s}$ & $5.6589 \mathrm{~m} / \mathrm{s}$ \\
Propeller rotation rate & $47.1239 \mathrm{rad} / \mathrm{s}(7.5 \mathrm{rps})$ & $9.3619 \mathrm{rad} / \mathrm{s}(89.4 \mathrm{rpm})$ \\
Froude number & 0.11850 & 0.11849 \\
Reynolds number & $9.874110^{6}$ & $1.258710^{9}$ \\
Wave period & $2.185 \mathrm{~s}$ & $11 \mathrm{~s}$ \\
Encountered wave period & $1.6433 \mathrm{~s}$ & $8.2738 \mathrm{~s}$ \\
Wave height & $15.79 \mathrm{~cm}$ & $4 \mathrm{~m}$ \\
\hline
\end{tabular}

the water surface, so any discrepancies in both measurement or simulation might result to having no ventilation at all.

\section{Forces}

Comparisons of the full-scale computation have been made with experiments for the dynamics of the predicted forces on a single blade and over the complete propeller. In general, the predicted FS signals of the forces are in satisfactory agreement with the extrapolated MS measurements. The single blade (SB) loads in pod reference frame are compared for the axial component Xp of the thrust in figure 6 . The FS signal is in red and the measurement in black. The computed signal changes rapidly with very short transient between the large time step $0.1 \mathrm{~s}$ and the adapted time step at time $249 \mathrm{~s}$ and follows perfectly the measurement. The maximum of thrust of the blade, about $180 \mathrm{kN}$, occurs around $3 T e / 4$ but with also a maximum of amplitude of about $90 \mathrm{kN}$. The minimum thrust, about $90 \mathrm{kN}$ is around $T e / 4$, with amplitude less than $20 \mathrm{kN}$ in the computation. The computed propeller thrust results from the summation of the blades thrust and is given with figure 7 with comparison to the thrust measured on the port-side (PS) propeller. The computed averaged value is $432 \mathrm{kN}$ compared with the measured $462 \mathrm{kN}$ ( $6 \%$ of relative difference). The computed and extrapolated experimental signals share more or less the same minima, about $352 \mathrm{kN}$, but the computed maxima, about $519 \mathrm{kN}$ are lower than those obtained in the measurements with a difference of about $60 \mathrm{kN}$ ( $12 \%$ of the averaged value). The lower predicted maxima of the total thrust was also expected

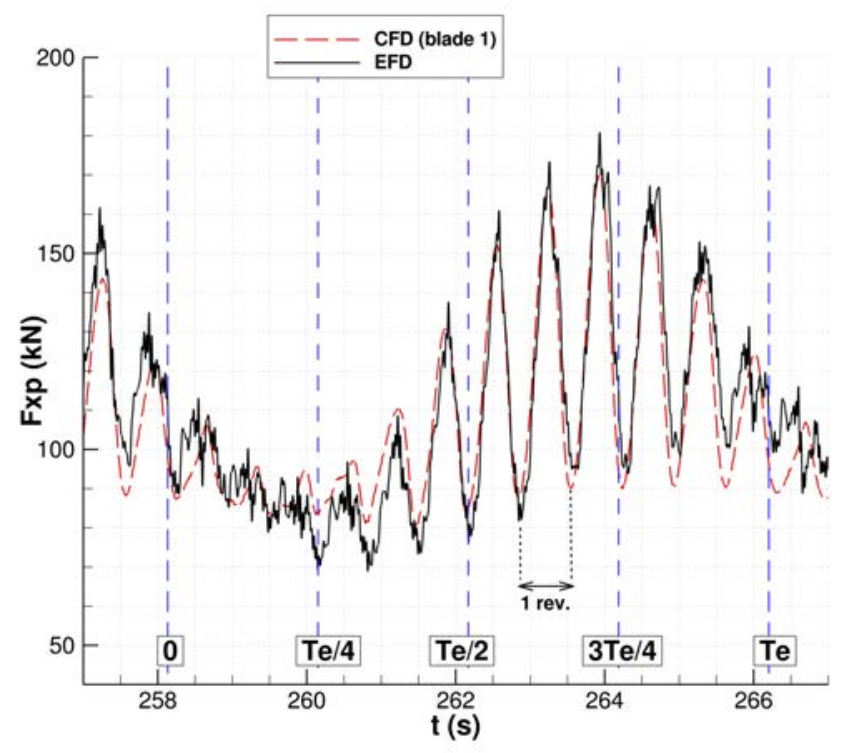

FIGURE 6. SINGLE BLADE THRUST IN POD REFERENCE FRAME. EFD: SOLID LINE, CFD: DASHED LINE.

from the single blade signal where the maxima are also lower than the measured maxima but not the minima. The contribution of each blade to the computed propeller thrust is hard to detect and the main behaviour is likely sinusoidal with the period of the encountered period Te. In experimenter's opinion, the high 


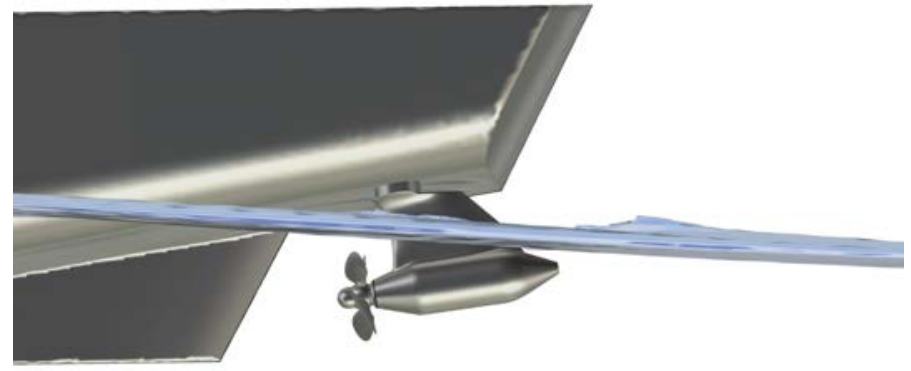

(a) $\mathrm{t}=\mathrm{t} 0=T e$

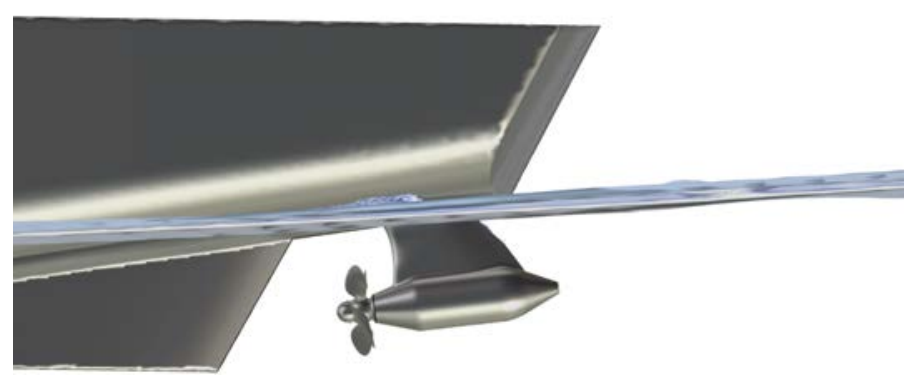

(c) $\mathrm{t}=\mathrm{t} 2=T e / 2$

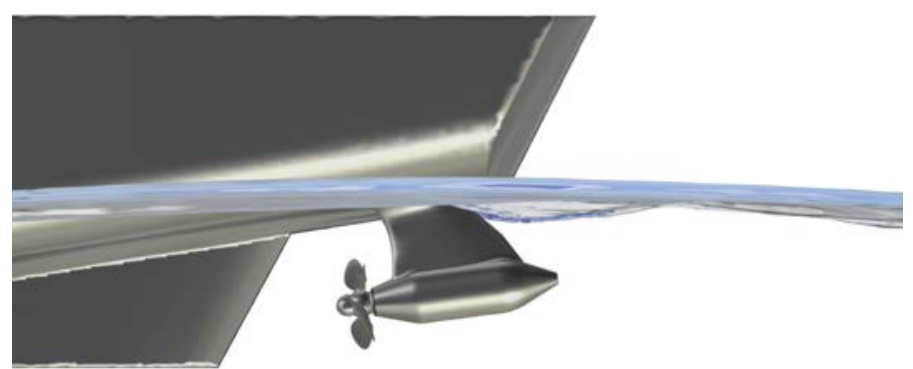

(b) $\mathrm{t}=\mathrm{t} 1=T e / 4$

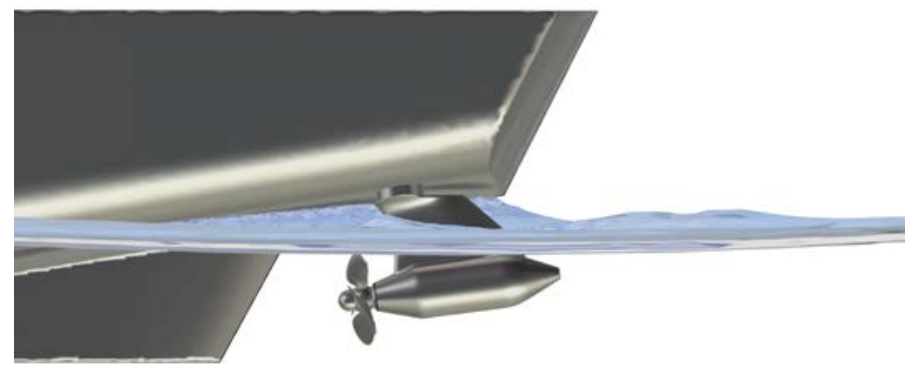

(d) $\mathrm{t}=\mathrm{t} 3=3 T e / 4$

FIGURE 4. INSTANTANEOUS FREE-SURFACE DEFORMATIONS AT FOUR PARTICULAR TIME INSTANTS DURING THE LAST COMPUTED ENCOUNTERED PERIOD $T e: 0, T e / 4, T e / 2$, AND 3Te/4. DETAILS IN THE PROPELLER REGION.

frequency oscillations in the experimented PS propeller signal may be due to vibrations coming from engine control artefacts. This also concerns the other components of the thrust and of the torque. As with the single blade, the minimum and maximum occur in the same time instants, at about Te/4 and 3Te/4, respectively.

The scale effect is focused on the normalized thrust coefficient $K_{T}\left(F x / \rho n D^{4}\right)$ of the axial Cartesian force component $F x$ from MS and FS simulations. The dynamic parameters used are those of Table 2 and the MS simulation is conducted in a manner similar to the FS simulation. The grid contains $5.15 \mathrm{M}$ cells (2.01M in the hull domain and 2.01M in the domain attached to the propeller) and is partitioned into 40 blocs. The time step in the initial phase is $0.025 \mathrm{~s}$ adapted to the wave propagation, and the second phase uses a time step $0.000666667 \mathrm{~s}$ adapted to the resolution of the propeller-hull-wave interactions. With regards to FS computation in the resolved step, the unsteady regime of the predicted MS signal of $K_{T}$ takes more time to establish. The scale effect on the thrust coefficient is weak on averaged values, about $4 \%$, with 0.179 in MS and $0.187(+0.008)$ in FS. In FS, the amplitude of the FS $K_{T}$ signal 0.0476 is lower than the MS amplitude signal 0.0541(-0.0065). The reason why the scale effect on the thrust is weak could be can explained by the fact that the propeller on the pod operates further from the hull than a screw propeller, then less sensitive to variation in the boundary layer thickness on the hull.

\section{Correlations}

The almost sinusoidal shape of the PS propeller thrust is due to the motion of the ship advancing in head wave and the way the effective wakefield changes just in front of the propeller during a wave encountered period. It is expected, like screw propeller, that the higher the velocity field in front of the propeller the lower the thrust produced by the propeller. Such a correlation has been established with the corresponding MS simulation carried out as a last test to evaluate the scale effect by probing the flow field during the computation in a slice in front of the propeller. Figure 8 collects the results of the post-process of the probes in this slice with the distribution of the normalized axial velocity component, the free-surface deformation and the ship in its computed position at the same four particular instants as used previously and selected from the pitch angle behaviour in time. The minimum value of the normalized velocity componenent is 0.827 at $\mathrm{t}=3 \mathrm{Te} / 4$ at the highest thrust, and the maximum is 1.028 at $\mathrm{t}=\mathrm{Te} / 4$ at the minimum thrust, while is it between the two at $\mathrm{t}=0=T e(0.943$, increasing velocity and decreasing thrust) and at $\mathrm{t}=\mathrm{Te} / 2$ (0.919, decreasing velocity and incresaing thrust). This confirms the expected behaviour of the dynamics of the axial thrust component relatively to the dynamics of the velocity 


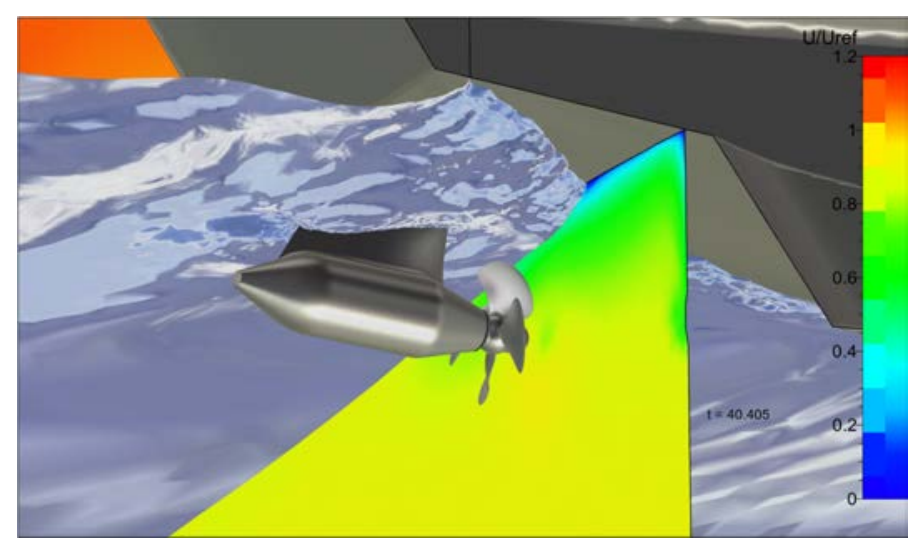

(a) $\mathrm{t}=\mathrm{t} 3=3 T e / 4$

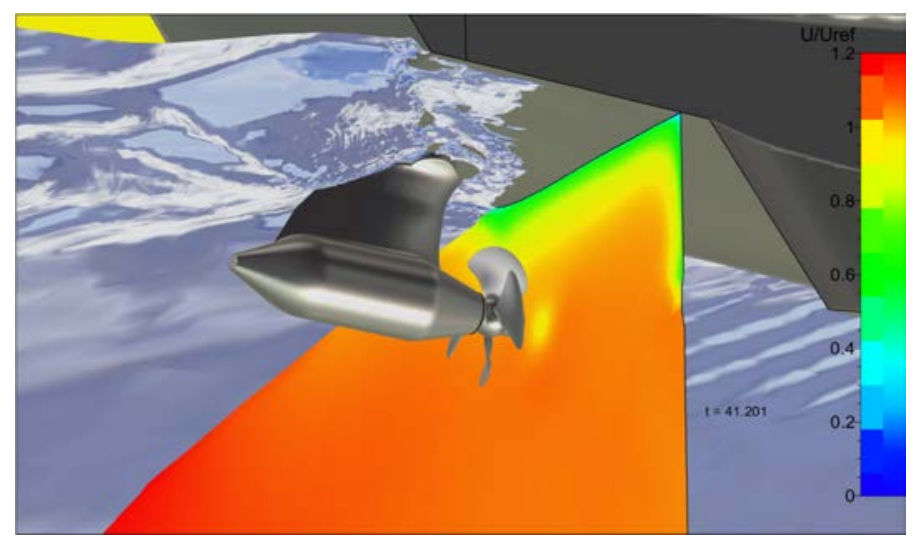

(c) $\mathrm{t}=\mathrm{t} 1=T e / 4$

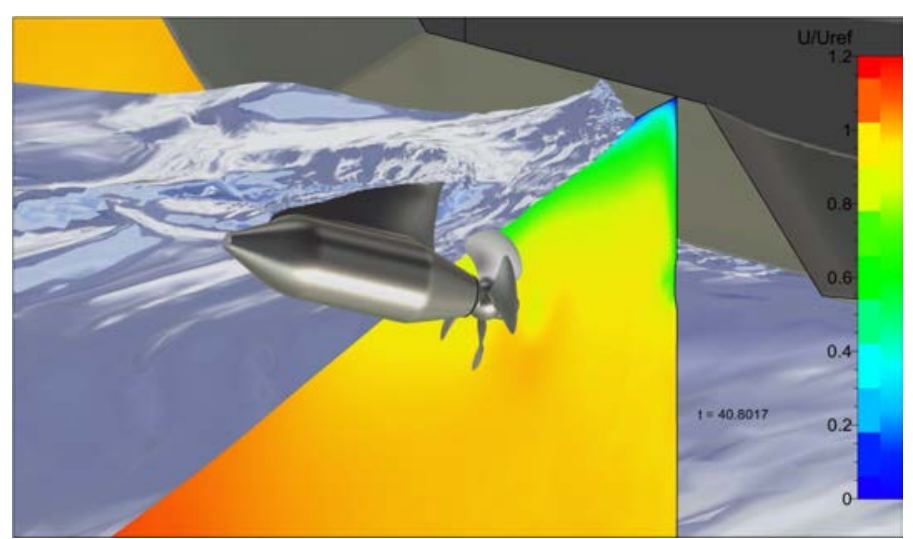

(b) $\mathrm{t}=\mathrm{t} 0=T e$

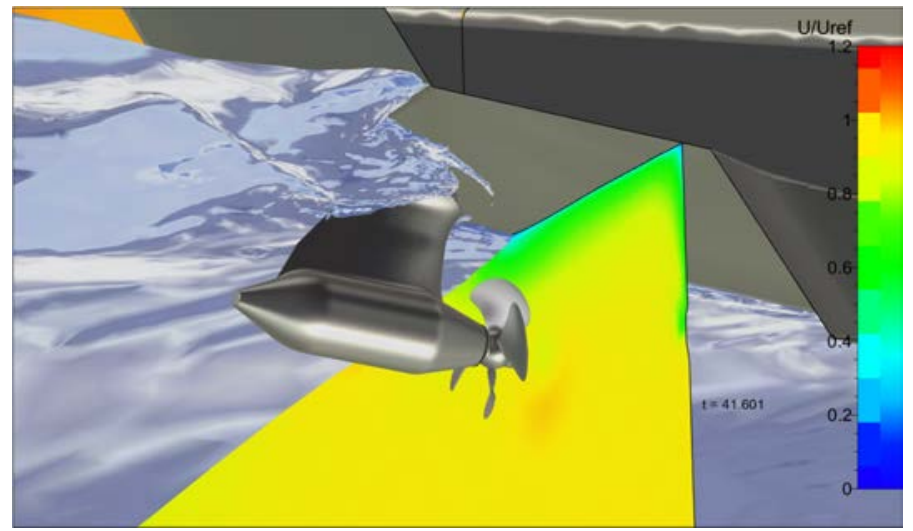

(d) $\mathrm{t}=\mathrm{t} 2=T e / 2$

FIGURE 8. FREE-SURFACE DEFORMATION AND DISTRIBUTION OF THE NORMALIZED CARTESIAN AXIAL VELOCITY COMPONENT AT FOUR PARTICULAR TIME INSTANTS OF THE THRUST DURING THE LAST COMPUTED WAVE PERIOD.

field in front of the propeller during a wave period.

\section{CONCLUSIONS}

The aim of the paper was to confront advanced computational and experimental fluid dynamics methods about the dynamic behaviour of the propeller loads for a podded ship in sailing condition. The conditions that are compared are very complicated. The model tests were the worlds first where a ship model was sailing in waves, while at the same time cavitation and ventilation were modelled and measured. The simulation of the podded propulsion of a ship advancing in head wave is quite challenging as it was the first time such a simulation was conducted with the considered flow solver. It involves interaction of the ship motions in waves with the rotating propeller and combines free-surface capturing, resolution of Newton's law to couple the forces induced by the flow and the motions of the ship, the sliding grid approach to deal with the rotating propeller, the mesh deformation technique to adapt the mesh to the ship motions including the rotating propeller. The work achieved was also at the leading edge of full RANS simulations conducted within the STREAMLINE project. On the considered computed case in atmospheric condition, no ventilation was detected neither in MS nor in FS conditions even if it was scarcely detected in wave basin. Is was outlined that this process is very sensitive to the exact height of the water surface but also water quality, so any discrepancies in both measurement or simulation might result to having no ventilation at all. The value of this case is not only on the absolute values of variables, as for ship's resistance, but on the dynamics involved. Considering the complex case and interactions, the agreement between the computed and measured thrusts is such that there is much more than a trend between experiments and computation. The computed flow field (both in direction and in intensity) entering the propeller is considered as accurate enough to consider the use of advanced CFD to get better understanding of flow phenomena and interactions for design improvements. 


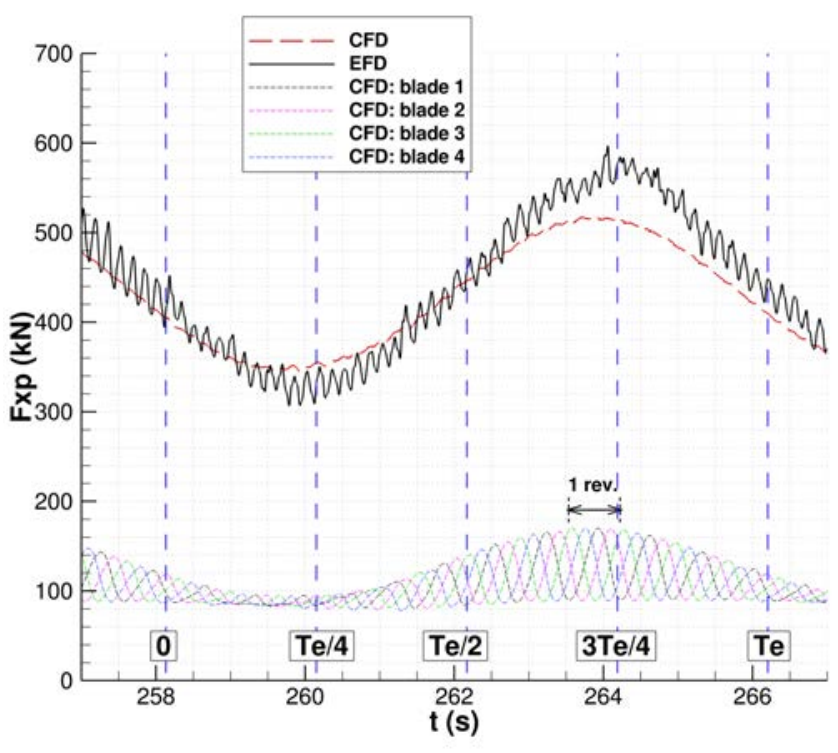

FIGURE 7. PROPELLER THRUST IN POD REFERENCE FRAME. EFD: SOLID LINE, CFD: DASHED LINE. OTHER THIN DASHED LINES FOR THE CFD THRUST OF EACH BLADE.

\section{ACKNOWLEDGMENT}

This work was granted access to the HPC resources of IDRIS under the allocation 2013-21308 made by GENCI (Grand Equipement National de Calcul Intensif).

Part of the work was supported by the STREAMLINE project, a collaborative R\&D project, partly funded by the 7 th Framework Programme of the European Commission (FP7).

\section{REFERENCES}

[1] Queutey, P., Deng, G. B., Guilmineau, E., and Salvatore, F., 2013. "A comparison between Full RANSE and coupled RANSE-BEM approaches in ship propulsion performance prediction". In Proceedings of 32nd International Conference on Ocean, Offshore and Artic Engineering, OMAE2013.

[2] Rijpkema, D., Starke, B., and Bosschers, J., 2013. "Numerical simulation of propeller-hull interaction and determination of the effective wake field using a hybrid RANS-BEM approach". In Proceedings of Third International Symposium on Marine Propulsors (SMP 2013).

[3] Deng, G., Queutey, P., Visonneau, M., and Salvatore, F., 2013. "Ship propulsion prediction with a coupled RANSEBEM approach.”. In Proceedings of the V International Conference on Computational Methods in Marine Engineering, MARINE-2013.

[4] Queutey, P., Deng, G., Wackers, J., and Visonneau, M., 2011. Ventilated propeller simulation with a RANSE solver. The 7th International Workshop on Ship Hydrodynamics (IWSH'2011).

[5] Carrica, P. M., Castro, A. M., and Stern, F., 2010. "Selfpropulsion computations using a speed controller and a discretized propeller with dynamic overset grids". Journal of Marine Science Technology, 15, pp. 316-330.

[6] Muscari, R., and Mascio, A. D., 2011. "Numerical simulation of the flow past a rotating propeller behind a hull". In 2nd International Symposium on Marine Propulsors, SMP 2011.

[7] Visonneau, M., Queutey, P., Deng, G. B., Wackers, J., Guilmineau, E., Leroyer, A., and Mallol, B., 2012. "Sliding grids and adaptive grid refinement for rans simulation of ship-propeller interaction.”. In 11th International Conference on Computer Applications and Information Technology in the Maritime Industries, COMPIT'12.

[8] Queutey, P., Deng, G. B., Wackers, J., Guilmineau, E., Leroyer, A., and Visonneau, M., 2012. "Sliding grids and adaptive grid refinement for rans simulation of shippropeller interaction". Ship Technology Research, Schiffstechnik, 59(2), pp. 44-58.

[9] Visonneau, M., Queutey, P., Wackers, J., Deng, G. B., and Guilmineau, E., 2012. "Sliding grids and adaptive grid refinement applied to ship hydrodynamics.”. In 29th ONR Symposium on Naval Hydrodynamics.

[10] Beek, T., v. Terwisga, T., and Bosschers, J., 2006. "Ventilation or cavitation: An experimental study to determine dynamic loads on controllable pitch propellers". In CAV2006.

[11] Lafeber, W., Brosset, L., and Bogaert, H., 2012. "Comparison of wave impact tests at large and full scale: Results from the sloshel project". In Proceedings of the Twentysecond International Offshore and Polar Engineering Conference,

[12] Hagesteijn, G., Brouwer, J., and Bosman, R., 2012. "Development of a six-component blade load measurement test set-up for propeller-ice impact". In Proceedings of 31nd International Conference on Ocean, Offshore and Artic Engineering, OMAE-2012.

[13] Queutey, P., and Visonneau, M., 2007. "An interface capturing method for free-surface hydrodynamic flows". Computers and Fluids, 36, pp. 1481-1510.

[14] Deng, G. D., and Visonneau, M., 1999. "Comparison of explicit algebraic stress models and second-order turbulence closures for steady flow around ships". In 7th Symposium on Numerical Ship Hydrodynamics, pp. 4.4-1-4.4-15.

[15] Duvigneau, R., Visonneau, M., and Deng, G. B., 2003. “On the role played by turbulence closures in hull ship optimization at model and full scale". Journal of Marine Science and Technology, 8, pp. 11-25.

[16] Leroyer, A., and Visonneau, M., 2005. "Numerical methods for RANSE simulations of a self-propelled fish-like body”. Journal of Fluids and Structures, 20, pp. 975-991. 